\title{
REVUE
}

\section{ÉTAT ACTUEL DU CONTROLE LAITIER EN FRANCE (1)}

\author{
par \\ M. LEROY
}

Les spécialistes admettent que pour les opérations de testage permettant de choisir les taureaux de reproduction destinés à l'insémination artificielle de l'ensemble de notre troupeau de vaches laitières, il faut pouvoir disposer d'un million de vaches soumises régulièrement au contrôle laitier. Or, ce nombre, qui n'était encore en 1964 que de 417000 têtes, s'est accru rapidement au cours des dernières années. Il était à la fin de 1967 de 726000 vaches, après avoir subi un accroissement de 36 p. 100 entre 1966 et 1967. Comme il est à présumer que ce mouvement va continuer, nous pouvons aisément prévoir que le million de vaches en contrôle sera atteint dans le courant de 1969. L'utilisation tant souhaitée de taureaux d'insémination, tous qualifiés par des épreuves prouvant les qualités laitières de leurs filles, entrera alonc rapidement dans la réalité.

A l'heure actuelle, presque tous les départements possèdent un organisme de contrôle laitier ( 80 sur 95 ). La moyenne des vaches contrôlées par troupeau est de 16 , alors qu'il n'était que de 13 vaches en 1964. Les effectifs de 10 à 20 vaches sont en majorité dans l'ensemble de la répartition, car ils représentent, en effet, 57,2 p. 100 du total. Le nombre des troupeaux soumis au contrôle est de 46175 . Parmi ceux-ci, près de 400 troupeaux dépassent un effectif de 50 vaches. Voici le détail de cette répartition :

EFFECTFS DE TROUPEAUX CONTROLÉs EN 1967

Moyenne générale 16 vaches par troupeau

\begin{tabular}{l|c|c|c|c|c}
\hline \hline $\begin{array}{l}\text { Moins de } \\
10 \text { vaches }\end{array}$ & $\begin{array}{c}\text { De } 10 \grave{a} \\
20 \text { vaches }\end{array}$ & $\begin{array}{c}\text { De 20 ̀ } \\
30 \text { vaches }\end{array}$ & $\begin{array}{c}\text { De } 30 \grave{a} \\
40 \text { vaches }\end{array}$ & $\begin{array}{c}\text { De } 40 \grave{a} \\
50 \text { vaches }\end{array}$ & $\begin{array}{c}\text { Plus de } \\
50 \text { vaches }\end{array}$ \\
\hline $20,4 \%$ & $57,2 \%$ & $16 \%$ & $4,1 \%$ & $1,3 \%$ & $0,8 \%$ \\
\hline \hline
\end{tabular}

(1) O. R. Acad. Agr., 1968, no 4, 242. 
Il faut faire justice de cette idée fausse qui prétend que le contrôle laitier ne peut convenir qu'aux propriétaires de grands troupeaux. Tous les possesseurs de vaches laitières devraient avoir l'obligation de soumettre leurs animaux au contrôle, car le bénéfice qu'ils peuvent en retirer dépasse de très loin le coût des opérations à effectuer.

L'extension considérable du contrôle laitier dans notre pays est due à l'heureuse initiative des services du Ministère des Finances, en prévision des dispositions de la récente loi sur l'élevage, des crédits importants pour renforcer les effectifs de contrôleurs des syndicats de contrôle laitier-beurrier et pour aider les syndicats on leur permettant de sortir de leurs coutumières difficultés financières. Il convient, en particulier, de féliciter M. Poly, Directeur de recherches de l'I.N.R.A., qui a été appelé en qualité de conseiller auprès du cabinet de M. Edgar Faure, et qui est en grande partie responsable de ces heureuses dispositions. De son côté, Mlle Sentix, secrétaire général du comité fédéral national de contrôle laitierbeurrier, qui a été la première à doter son organisation d'un matériel mécanographique ayant longtemps servi d'exemple aux groupements européens poursuivant le même objet, assure sans défaillances au prix d'efforts méritoires, la difficile coordination de l'ensemble des travaux effectués sur notre territoire.

Rappelons que le contrôle laitier-beurrier est effectué chaque mois avec l'aide de contrôleurs, engagés par des syndicats d'éleveurs. Les opérations effectuées par ces contrôleurs sont codifiées d'après un texte élaboré par des experts réunis sur l'initiative à la FAO, à Rome. L'application de ce texte dans le cadre européen est surveillée par le Comité européen du Contrôle laitier-beurrier. Les dispositions du texte primitif ont été reprises par l'organisation internationale de standardisation (I.S.O.), et font partie d'une recommandation de cet organisme qui porte le $\mathrm{n}^{\circ} \mathbf{1 5 4 6 .}$

La dernière publication du Comité Fédéral national français de contrôle laitier-beurrier donne 407000 résultats concernant les vaches ayant terminé leur lactation au cours de l'année 1966. Elle nous renseigne sur la répartition des animaux contrôlés dans chaque département, sur les races auxquelles appartiennent ces animaux, et sur les performances laitières-beurrières moyennes classées par race. On peut voir ainsi qu'un certain nombre de départements, en tête desquels il faut citer l'Orne, l'Aisne et la Mayenne, possèdent plus de 15000 vaches soumises au contrôle.

Par rapport à leur effectif recensé de vaches laitières, l'Aisne, les Ardennes, la Mayenne et la Meuse possèdent plus de 20 p. 100 d'animaux contrôlés par rapport à l'effectif des vaches du troupeau départemental. Quelques autres départements, tels que le Doubs, la Marne, la Moselle et l'Orne, sont bien près d'atteindre le même résultat. En revanche, les départements ayant les plus forts effectifs de bovins, lesquels, à part le Nord, font partie de la pénin- 
sule armoricaine et de la Normandie, ne contrôlent qu'à peine la dixième partie de leur effectif, ce qui est notoirement insuffisant et donne la mesure des progrès encore à réaliser.

Toutes les races laitières françaises, qui sont au nombre de 16 , figurent parmi les vaches contrôlées. Mais les 3 principales d'entre elles, la Frisonne pie-noire, la Normande et le groupe des Pierouge (Montbéliarde, Tachetée de l'Est, Abondance) comptent près de 90 p. 100 de l'ensemble $(88,5$ p. 100$)$.

Pour 1966, la race Frisonne pie-noire vient en tête, avec 190700 vaches. La moyenne individuelle des rendements enregistrés est de $3760 \mathrm{~kg}$, avec une teneur en matière grasse de $3,62 \mathrm{p}$. 100, pour une lactation d'une durée moyenne de 281 jours.

Sur eet effectif, 1800 vaches ont fourni en une lactation plus de $7000 \mathrm{~kg}$, et 174 ont dépassé le niveau annuel de $9000 \mathrm{~kg}$. Ces résultats expliquent pour quelle raison cette race compte des représentants dans 62 départements, et s'étend progressivement en Bretagne et dans certains départements normands, notamment dans l'Eure, la Seine-Maritime et l'Orne.

La race Normande se classe après la Frisonne, en ce qui concerne l'effectif contrôlé la même année, qui est de 133000 têtes, comme en ce qui regarde les performances moyennes réalisées, qui sont de $3229 \mathrm{~kg}$ de lait à 4,03 p. 100 de taux butyreux, pour une durée de lactation de 277 jours. D'après les résultats obtenus, provenant de 37 départements, les génisses normandes mettent bas la première fois un peu plus tard que les Frisonnes, et donnent au cours de leur première lactation un peu moins de lait, par comparaison avec les rendements fournis par les vaches adultes. Le tableau ci-après fourni des renseignements détaillés sur ces différences.

COMPARATSON DES PERTORMANCES PAR LACTATION DES NORMANDAS ET DES FRISONNES, POUR LA CAMPAGNE EN 1965-1966

\begin{tabular}{|c|c|c|}
\hline & $\begin{array}{c}\text { Race française } \\
\text { Frisonne }\end{array}$ & Race Normande \\
\hline Age au premier vêlage . . . . . . . . . & 2 ans 9 mois & 2 ans 11 mois \\
\hline Première lactation ............. & $3181 \mathrm{~kg} 76,7$ & $2647 \mathrm{~kg} 73,5$ \\
\hline 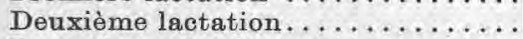 & $3586 \mathrm{~kg} 86,0$ & $2953 \mathrm{~kg} 82,0$ \\
\hline Lactations ultérieures............ & $4157 \mathrm{~kg} 100$ & $3604 \mathrm{~kg} 100$. \\
\hline
\end{tabular}

La concurrence entre ces deux races est, pour ces raisons, très forte, et l'on peut affirmer qu'elle a été et qu'elle demeure encore l'un des facteurs les plus importants de l'accroissement de la production laitière française.

Lorsque l'on examine d'un département à l'autre, les résultats fournis par les vaches de ces deux races, il est frappant de considérer 
les écarts qui existent entre les sujets vivant dans ce qu'il convient d'appeler les berceaux de la race, par rapport à ceux des régions où ces animaux ont été introduits plus tardivement. Ainsi, par exemple, si l'on considère les Frisonnes, le rendement moyen des femelles de cette race dans les départements du berceau dépasse légèrement $4000 \mathrm{~kg}$ avec un taux butyreux de 3,60 p. 100 alors que celui des vaches frisonnes du sud-ouest et du centre est souvent au-dessous de $3500 \mathrm{~kg}$ avec un taux butyreux qui ne dépasse pas 3,40 p. 100. Il existe cependant d'heureuses exceptions, comme celles de l'Eure-et-Loir et du Loir-et-Cher, où les performances des Frisonnes contrôlées dépassent même celles de leurs homologues de l'Aisne et du Nord (4 220 kg).

Les mêmes observations peuvent être faites en ce qui concerne la race normande. Les vaches contrôlées dans les einq départements normands fournissent à l'âge adulte $3800 \mathrm{~kg}$ de lait à $4,03 \mathrm{p} .100$, tandis que celles de la Charente, la Charente-Maritime, le Finistère et l'Tlle-et-Vilaine ne donnent dans les mêmes conditions que $3200 \mathrm{~kg}$, ee qui contribue fortement à la diminution de la moyenne générale pour la race.

Cette observation mérite de retenir l'attention. En effet, la vulgarisation de la féeondation artificielle dans les régions en dehors du berceau a permis l'introduction dans les Centres d'insémination de reproducteurs de grande valeur souvent achetés très cher, dont la semence a été fort généreusement répartie. On peut done s'étonner que cette amélioration du niveau génétique des populations bovines n'ait pas été suivie par une hausse plus sensible de la production laitière individuelle. L'explication qui en découle est que les notions de l'alimentation rationnelle des troupeaux n'ont pas été suffisamment généralisées, tant par les agents chargés de la vulgarisation que par les inséminateurs et les contrôleurs laitiers. Il nous paraît indispensable de créer un corps spécial d'agents capables d'enseigner aux éleveurs les méthodes à utiliser pour alimenter correctement les bonnes laitières, surtout pendant la mauvaise saison, et au cours des premiers mois de lactation. Ce n'est qu'à ce prix que les taureaux sélectionnés par le testage seront en mesure de donner dans l'avenir tout ce que l'on peut attendre d'eux.

La diffusion des épreuves de contrôle laitier dans nos départements des Vosges, du Jura et des Alpes a été relativement lente et tardive. C'est la raison pour laquelle le groupe des Pie-rouge de l'Est, dont l'effectif est sensiblement égal à celui de la race Normande, compte relativement peu de vaches contrôlées. L'effectif de ces races n'est, en effet, en 1966 que de 36200 têtes, dont 17000 Montbéliardes, 11000 Tachetées et 8200 Abondance.

Mais il convient de mentionner toutefois les progrès remarquables accomplis dans les départements du Doubs et du Jura, où les vaches 
laitières, dont la production dépasse $4000 \mathrm{~kg}$, se rencontrent maintenant d'une manière courante.

Parmi les autres races, nous n'aurons de garde d'omettre la Brune des Alpes, qui fait actuellement de très grands progrès dans l'Ariège, l'Aude, l'Aveyron et le Tarn et qui compte près de 10000 vaches au contrôle. Dans son berceau français, situé aux confins des départements de la Côte-d'Or, de l'Aube et de l'Yonne, le rendement moyen par lactation est d'environ $3600 \mathrm{~kg}$, compte tenu des vaches en première et deuxième lactation, ce qui correspond à une production de plus de $4000 \mathrm{~kg}$ par vache adulte. Le taux butyreux de cette race est de 3,60 p. 100 et entièrement comparable à coux des Montbéliardes et des Tachetées de l'Est.

Il nous reste, pour achever ce tableau de l'extension du contrôle laitier dans notre pays, à mentionner la parfaite réussite des efforts entrepris pour introduire chez nous le contrôle de la matière azotée du lait. Tant au point de vue de l'hygiène de l'homme que de l'économie de la production fromagère, l'augmentation du taux de la matière azotée dans le lait de vache est souhaitable.

Bien que liée dans une certaine mesure au taux butyreux, la matière azotée, pour des teneurs en matière grasse identiques, telles, par exemple, de 4 p 100, peut varier entre 2,70 et 4 p. 100 , selon les individus. D'autre part, la possibilité d'obtenir par sélection des souches de bétail laitier caractérisées par de hautes teneurs en matière grasse et en caséine a été amplement démontrée.

Fort heureusement, notre pays, après les Pays-Bas, a été l'un des premiers à compléter le contrôle beurrier par le contrôle de la matière azotée. Le nombre des vaches actuellement soumis au double contrôle laitier atteindra cette année 150000 . En raison de la moindre variation du taux de matière azotée, par rapport à celle que l'on observe couramment pour le taux de matière grasse, le nombre d'échantillons à prélever par lactation n'est pas aussi grand que pour le contrôle beurrier.

Les échantillons, conservés au moyen de bichlorure de mercure, sont adressés à des laboratoires spécialisés, qui travaillent sous la surveillance du département de microbiologie et de l'industrie laitière de l'I.N.R.A., dirigé par M. Mocquot. Ils sont analysés par une méthode colorimétrique dite au noir amido, qui est basée sur l'absorption d'une matière colorante spéciale par les matières protéiques du lait. Cette méthode est sensible et délicate. Elle exige un appareillage spécial et doit être périodiquement vérifiée par des contre-analyses effectuées par la méthode de Kjeldahl.

Des résultats fort prometteurs ont déjà été obtenus. Dans le département de l'Aisne, notamment, de grandes différences ont été constatées sur des taureaux en ce qui concerne leur aptitude à fournir de la matière azotée à leurs filles. On ne peut qu'attendre de cette méthode de très nombreux et très intéressants progrès. 